CLINICAL STUDY

\title{
Inflammatory and endothelial dysfunction markers and proteinuria in persons with type 1 diabetes mellitus
}

\author{
Karine Sahakyan, Barbara E K Klein, Kristine E Lee, Michael Y Tsai ${ }^{1}$ and Ronald Klein \\ Department of Ophthalmology and Visual Sciences, University of Wisconsin School of Medicine and Public Health, 610 North Walnut Street, \\ Fourth Floor WARF, Madison, Wisconsin 53726, USA and ${ }^{1}$ Department of Laboratory Medicine and Pathology, University of Minnesota Medical School, \\ 420 Delaware Street SE, Minneapolis, Minnesota 55455, USA \\ (Correspondence should be addressed to K Sahakyan; Email: sahakyan@epi.ophth.wisc.edu)
}

\begin{abstract}
Objective: We examined the relationship of inflammatory and endothelial dysfunction markers with the prevalence and incidence of gross proteinuria (GP) in persons with type 1 diabetes mellitus.

Design: A longitudinal population-based cohort of persons with type 1 diabetes mellitus was followed from 1990-1992 through 2005-2007.

Methods: Prevalence and 15-year cumulative incidence of GP were defined as outcome variables. Serum high-sensitivity C-reactive protein (hsCRP), interleukin-6 (IL-6), tumor necrosis factor- $\alpha$ (TNF- $\alpha$ ), soluble vascular cell adhesion molecule-1 (VCAM-1), soluble intercellular adhesion molecule-1, and serum total homocysteine were measured. Multivariate logistic and discrete linear logistic regression modeling was used for data analysis.

Results: After controlling for duration of diabetes and other confounding factors, TNF- $\alpha$ (odds ratio (OR) 3.64; 95\% confidence interval (CI) 2.33, 5.70), IL-6 (OR 1.41; 95\% CI 1.06, 1.88), VCAM-1 (OR 13.35; 95\% CI 5.39, 33.07), and homocysteine (OR 2.98; 95\% CI 1.73, 5.16) were associated with prevalent proteinuria. Only hsCRP (OR 1.47; 95\% CI 1.02, 2.11) was associated with incident proteinuria.

Conclusions: These findings suggest a role of inflammation and endothelial dysfunction as markers and contributors of the development of diabetic nephropathy in persons with type 1 diabetes mellitus.
\end{abstract}

European Journal of Endocrinology 162 1101-1105

\section{Introduction}

Nephropathy contributes to excess morbidity and mortality in people with diabetes $(1,2)$. A number of factors such as hyperglycemia, longer diabetes duration, high blood pressure, and high serum lipid levels have been shown to be associated with the development and progression of diabetic nephropathy (1-4). However, they explain only a limited amount of risk of developing diabetic nephropathy.

Inflammation and endothelial dysfunction have been hypothesized to play a role in the pathogenesis of diabetic nephropathy (5-8). However, only one population-based study described the relationship of inflammatory and endothelial dysfunction markers to the incidence of nephropathy in persons with type 1 diabetes mellitus (8). Thus, it is not clear whether inflammation and/or endothelial dysfunction are primary consequences of the progression of diabetic nephropathy or contributors to it. The purpose of our study was to investigate the association between inflammatory and endothelial dysfunction markers and the prevalence and 15-year cumulative incidence of gross proteinuria (GP) in a cohort of persons with type 1 diabetes mellitus.

\section{Materials and methods}

\section{Study population}

The population consisted of a sample of 1210 persons with type 1 diabetes mellitus who received primary care in an area of 11 counties in Southern Wisconsin from 1979 to $1980(9,10)$. Of these, 996 participated in the baseline examination (1980-1982), 903 in the 4-year follow-up (1984-1986), 816 in the 10-year follow-up (1990-1992), 667 in the 14-year follow-up (1994-1996), 567 in the 20-year follow-up (20002002), and 520 in the 25-year follow-up (2005-2007) (9-12). The main reason for nonparticipation was death (9-12). Comparisons between participants and nonparticipants at the various examinations have been presented elsewhere (9-12). Frozen serum was 
available from the time of the 10-year examination in 1990-1992. Analyses in this report are limited to 795 persons who attended the 10-year examination, had frozen serum available from that examination, and had information about proteinuria at the 14-year (1994-1996) and/or 20-year (2000-2002) and/or 25-year (2005-2007) follow-up examinations.

\section{Procedures}

The examinations were performed in a mobile examination van in or near the city where the participants resided. Informed consent was obtained from participants, and all examinations followed a similar protocol that was approved by the institutional Human Subjects Committee of the University of Wisconsin and conformed to the Declaration of Helsinki. A structured interview and examinations were conducted by trained examiners.

At the time of the 10-year follow-up examination, an aliquot of whole blood was used for determination of the HbAlc level using the Quik-SEP Fast Hemoglobin affinity chromatography test system (Isolab Inc., Akron, OH, USA). The normal range for HbAlc was 4.6-7.9\%, and its intra-assay coefficient of variation was $2.4 \%$. Serum was used to measure total and high-density lipoprotein cholesterol. The remaining serum for 674 persons was stored without preservative at $-80{ }^{\circ} \mathrm{C}$ in cryogenic vials with O-rings for up to 16 years until the vials were shipped on dry ice to the University of Minnesota laboratory for the analyses reported herein. An aliquot of serum was used for determination of high-sensitivity C-reactive protein (hsCRP), interleukin-6 (IL-6), tumor necrosis factor- $\alpha$ (TNF- $\alpha$ ), endothelial dysfunction markers (soluble vascular cell adhesion molecule-1 (VCAM-1) and soluble intercellular adhesion molecule1 (ICAM-1)), and serum total homocysteine.

The level of hsCRP was measured on the Hitachi 911 (Roche Diagnostics) using the CRP K-Assay, a particleenhanced immunonephelometric assay (Kamiya Biomedical Company, Seattle, WA, USA). IL- 6 , TNF- $\alpha$, VCAM-1, and ICAM-1 were measured by ELISA assays employing quantitative sandwich enzyme immunoassay techniques. IL- 6 was measured using the Quantikine HS Human IL-6 Immunoassay Kit (R\&D Systems, Minneapolis, MN, USA). TNF- $\alpha$ was measured using the QuantiGlo Chemiluminescent ELISA kit (R\&D Systems). Soluble VCAM-1 was measured using the Quantikine Human sVCAM-1 Immunoassay kit (R\&D Systems). Soluble ICAM-1 was measured using the Quantikine Human sICAM-1 Immunoassay kit (R\&D Systems). Serum total homocysteine was measured by a fluorescence polarization immunoassay (IMx Homocysteine Assay, Axis Biochemicals ASA, Oslo, Norway) using the IMx Analyzer (Abbott Diagnostics).

Single voided, casual, fresh urine samples were collected for the determination of proteinuria at all examinations; GP was measured using a reagent strip (Labstix, Ames Division, Miles Inc., Elkhart, IN, USA).

\section{Definitions}

We defined GP as having urinary protein concentrations of $>0.3 \mathrm{~g} / \mathrm{l}$. Cumulative incidence of GP was defined as being present at any follow-up examination in persons in whom it was absent at the 1990-1992 Wisconsin Epidemiologic Study of Diabetic Retinopathy (WESDR) examination. Persons were categorized as non-smokers, ex-smokers, or current smokers. Hypertension status was defined as normotensive if a participant did not have a history of hypertension and the systolic blood pressure was $<140 \mathrm{mmHg}$ and diastolic blood pressure was $<90 \mathrm{mmHg}$, as hypertensive with blood pressure controlled if a participant had a history of hypertension and the systolic blood pressure was $<140 \mathrm{mmHg}$ and diastolic blood pressure was $<90 \mathrm{mmHg}$, and as hypertensive with blood pressure uncontrolled if a participant had a history of hypertension and the systolic blood pressure was $\geq 140 \mathrm{mmHg}$ and/or diastolic blood pressure was $\geq 90 \mathrm{mmHg}$. Age was defined as the age at the time of the 1990-1992 examination. The duration of diabetes was that period between the age at diagnosis and the age at the 1990-1992 examination. BMI was defined as weight in $\mathrm{kg}$ divided by height in $\mathrm{m}^{2}$. Total family income was defined as low $(<\$ 30000 /$ year $)$ or not low ( $\geq \$ 30000 /$ year) at the 1990-1992 examination. A person was defined as having a history of cardiovascular disease, ketoacidosis, or neuropathy at the 1990-1992 examination based on a history of these conditions. Cardiovascular disease history was defined based on the participant's history of angina, myocardial infarction, or stroke. Neuropathy was defined as either a self-reported history of loss of tactile or temperature sensitivity or both. Severity of retinopathy was determined by grading of the fundus photographs by means of a modified Airlie House classification scheme as further adapted for the WESDR follow-up examinations (13).

\section{Statistical methods}

The distributions of the inflammatory and endothelial dysfunction markers were highly skewed. Therefore, we performed logarithmic transformation of these variables to use as a continuous exposure variable in the analyses.

Statistical methods included calculations of means and frequencies and performing multivariate logistic regression and discrete linear logistic regression analysis. Age, gender, total family income, diabetes duration, hypertension, history of neuropathy, serum total cholesterol, and HbAlc levels were considered confounding variables. Only one inflammatory or endothelial dysfunction marker was included in each model because they were highly correlated. Relationships of inflammation and endothelial dysfunction markers to the prevalence or incidence of GP were examined in separate multivariate logistic regression 
models. Cumulative 15-year incidence of GP was determined using the Kaplan-Meier approach accounting for the competing risk of death; relation of inflammation and endothelial dysfunction markers to incident GP was determined using a proportional hazards approach with discrete linear logistic regression modeling. Proportional hazards assumption was tested for all models. Stratified or time-dependent Cox regression analyses were performed to adjust for assumed violation of proportional hazards assumption. All models were tested for multicollinearity and interaction.

\section{Results}

Of the 795 people examined at the 1990-1992 baseline examination, 744 had information on proteinuria status, and $201(27.0 \%)$ had GP. Of the 543 people without proteinuria, $112(20.6 \%)$ were alive but not examined at follow-up due to refusal or moving out of area and unable to return for an examination, and 47 (8.7\%) died, leaving 384 (70.7\%) people for incidence analysis.
Characteristics of the population by GP status at the baseline examination are presented in Table 1. People with GP at baseline had a longer duration of diabetes, higher mean HbAlc, and serum total cholesterol levels, were more likely to be male, have lower income, and have more frequent history of ketoacidosis and neuropathy than those without GP (Table 1). Additionally, people with GP had higher levels of all inflammatory or endothelial dysfunction markers than those without GP, except for hsCRP. People without GP had a lower frequency of history of cardiovascular disease and uncontrolled hypertension. We did not find any associations of age, BMI, or smoking status with GP.

Analysis of cross-sectional associations between the levels of inflammatory and endothelial dysfunction markers and GP is shown in Table 2. Multivariate logistic analysis revealed that serum TNF- $\alpha$, IL-6, VCAM-1, and homocysteine levels were cross-sectionally associated with GP (Table 2).

The 15-year cumulative incidence of GP was $24.4 \%$. After controlling for age and other confounding variables, only serum hsCRP was significantly associated with incident GP (Table 2).

Table 1 Baseline characteristics by gross proteinuria status at the 1990-1992 Wisconsin Epidemiologic Study of Diabetic Retinopathy examination.

\begin{tabular}{|c|c|c|c|}
\hline Characteristic & $\begin{array}{l}\text { Gross proteinuria } \\
\text { present }(n=201)^{\mathrm{a}}\end{array}$ & $\begin{array}{l}\text { No proteinuria } \\
\qquad(n=543)^{\mathrm{a}}\end{array}$ & $P$ value \\
\hline $\begin{array}{l}\text { Age (years), mean } \pm \text { s.D. } \\
\text { Males, } n(\%)\end{array}$ & $\begin{array}{l}37.5 \pm 11.0 \\
115(57.2)\end{array}$ & $\begin{array}{r}36.8 \pm 11.9 \\
249(49.5)\end{array}$ & $\begin{array}{l}0.5 \\
0.006\end{array}$ \\
\hline \multicolumn{4}{|l|}{ Family income, $n(\%)$} \\
\hline$<\$ 30000$ & $109(57.1)$ & $229(47.4)$ & 0.02 \\
\hline \multicolumn{4}{|l|}{$\mathrm{BMI}, n(\%)$} \\
\hline $25-29 \mathrm{~kg} / \mathrm{m}^{2}$ & $69(37.5)$ & $212(42.0)$ & 0.6 \\
\hline$\geq 30 \mathrm{~kg} / \mathrm{m}^{2}$ & $26(14.1)$ & 65 (12.9) & \\
\hline \multicolumn{4}{|l|}{ Smoking history, $n(\%)$} \\
\hline Ex-smoker & $48(24.1)$ & $120(22.2)$ & 0.8 \\
\hline Current smoker & $42(21.1)$ & $112(20.7)$ & \\
\hline Diabetes duration (years), mean \pm s.D. & $23.8 \pm 8.5$ & $22.4 \pm 9.6$ & 0.06 \\
\hline HbAlc (\%), mean \pm S.D. & $10.5 \pm 1.7$ & $9.8 \pm 1.7$ & $<0.001$ \\
\hline \multicolumn{3}{|l|}{ Retinopathy history, $n(\%)$} & $<0.0001$ \\
\hline Mild & $33(16.7)$ & $261(48.2)$ & \\
\hline Moderate & $30(15.2)$ & $96(17.7)$ & \\
\hline Proliferative diabetic retinopathy & $134(67.7)$ & $152(28.1)$ & \\
\hline Neuropathy history, $n(\%)$ & $80(39.8)$ & $116(21.4)$ & $<0.001$ \\
\hline Ketoacidosis history, $n(\%)$ & $33(16.7)$ & $52(9.65)$ & 0.009 \\
\hline Hypertension BP controlled, $n(\%)$ & $58(30.4)$ & $59(11.7)$ & $<0.001$ \\
\hline Hypertension BP uncontrolled, $n(\%)$ & $39(20.4)$ & 29 (5.77) & \\
\hline Cardiovascular disease history, $n(\%)$ & $23(11.7)$ & $39(7.22)$ & 0.05 \\
\hline \multicolumn{4}{|l|}{ Serum } \\
\hline hsCRP $\left(\mathrm{mg} / \mathrm{l}^{\mathrm{b}}\right.$ & $1.6(3.5-120.2)$ & $1.4(2.80-174.7)$ & 0.11 \\
\hline $\mathrm{IL}-6(\mathrm{pg} / \mathrm{ml})^{\mathrm{b}}$ & $1.9(1.8-53.9)$ & $1.5(1.33-78.4)$ & $<0.0001$ \\
\hline $\operatorname{VCAM}-1(\mathrm{ng} / \mathrm{ml})^{\mathrm{b}}$ & $945.8(328.3-1866)$ & $806.3(255.0-1189.0)$ & $<0.0001$ \\
\hline ICAM-1 (ng/ml) & $306.2(72.4-397.9)$ & $298.5(81.8-662.6)$ & 0.02 \\
\hline TNF- $\alpha(\mathrm{pg} / \mathrm{ml})^{\mathrm{b}}$ & $3.1(2.2-9.6)$ & $1.9(1.3-26.3)$ & $<0.0001$ \\
\hline Homocysteine $(\mu \mathrm{mol} / \mathrm{l})^{\mathrm{b}}$ & $12.5(6.5-62.1)$ & $9.0(3.8-37.4)$ & $<0.0001$ \\
\hline
\end{tabular}


Table 2 Relationship of inflammatory and endothelial dysfunction markers and prevalence and incidence of gross proteinuria at the 1990-1992 Wisconsin Epidemiologic Study of Diabetic Retinopathy examination.

\begin{tabular}{|c|c|c|c|c|}
\hline \multirow[b]{2}{*}{ Markers $^{a}$} & \multicolumn{2}{|c|}{ Prevalent gross proteinuria } & \multicolumn{2}{|c|}{ Incident gross proteinuria } \\
\hline & Crude OR $(95 \% \mathrm{Cl})$ & Adjusted $\mathrm{OR}^{\mathrm{b}}(95 \% \mathrm{Cl})$ & Crude OR $(95 \% \mathrm{Cl})$ & Adjusted $\mathrm{OR}^{\mathrm{b}}(95 \% \mathrm{Cl})$ \\
\hline hsCRP (mg/l) & $1.12(0.97,1.29)$ & $1.04(0.87,1.25)$ & $1.41(1.06,1.86)$ & $1.47(1.02,2.11)$ \\
\hline IL-6 (pg/ml) & $1.54(1.23,1.94)$ & $1.41(1.06,1.88)$ & $1.47(1.05,2.05)$ & $1.44(0.87,2.39)$ \\
\hline VCAM-1 (ng/ml) & $15.82(1.71,33.30)$ & $13.35(5.39,33.07)$ & $4.96(1.28,19.25)$ & $4.62(0.98,21.67)$ \\
\hline ICAM-1 (ng/ml) & $2.35(1.08,5.13)$ & $1.80(0.72,4.46)$ & $3.32(1.07,10.28)$ & $1.40(0.28,7.05)$ \\
\hline $\mathrm{TNF}-\alpha(\mathrm{pg} / \mathrm{ml})$ & $4.62(3.19,6.71)$ & $3.64(2.33,5.70)$ & $1.62(1.02,2.57)$ & $1.57(0.77,3.19)$ \\
\hline Homocysteine $(\mu \mathrm{mol} / \mathrm{l})$ & $5.25(3.40,8.13)$ & $2.98(1.73,5.16)$ & $2.23(1.16,4.28)$ & $2.41(0.75,7.78)$ \\
\hline
\end{tabular}

OR, odds ratio; $\mathrm{CI}$, confidence interval; hsCRP, high-sensitivity C-reactive protein; IL-6, interleukin-6; VCAM-1, soluble vascular cell adhesion molecule-1; ICAM-1, soluble intercellular adhesion molecule-1; TNF- $\alpha$, tumor necrosis factor- $\alpha$.

${ }^{a}$ All markers are entered in the model using logarithmic transformation of continuous exposure variable.

${ }^{b}$ Controlling for age, gender, total family income, diabetes duration, comorbidities (history of hypertension and neuropathy history), serum total cholesterol, and HbAlc levels.

\section{Discussion}

While controlling for age and other factors, we found statistically significant cross-sectional associations of serum TNF- $\alpha$, IL- 6 , VCAM-1, and homocysteine with GP and of hsCRP with the 15-year cumulative incidence of GP in persons with type 1 diabetes mellitus. Our findings are consistent with previously reported crosssectional associations of inflammatory and endothelial dysfunction markers with nephropathy in persons with type 1 and type 2 diabetes mellitus $(5,6,14)$.

While controlling for other factors, all the inflammatory and endothelial dysfunction markers had higher odds of developing GP; however, only the association of serum CRP with GP was statistically significant in our study. A similar longitudinal association of hsCRP with urinary albumin excretion was reported in patients with type 2 diabetes mellitus (15). High levels of circulating hsCRP may deposit in the glomerular endothelium and cytoplasm of tubules. It is not clear whether such deposits result in damage to the glomerulus and interstitial fibrosis $(16,17)$. We speculate that the inflammatory process may be a result of and be involved in the pathogenesis of nephropathy in persons with type 1 diabetes mellitus. Circulating hsCRP may be an indicator of inflammation and endothelial cell activation in these patients.

While controlling for other risk factors, serum homocysteine was statistically significantly associated only with higher odds of prevalent GP. This is consistent with the finding that higher homocysteine levels are cross-sectionally associated with higher urinary albumin excretion rates $(18,19)$. However, data from experimental and observational studies showed no evidence of relationship of homocysteine level and progression of nephropathy (20). These findings suggest that elevated serum homocysteine levels are more likely a result of diabetic nephropathy than a cause.

While our study has many strengths, including the long-term follow-up, high participation rate of a wellcharacterized cohort of persons with type 1 diabetes mellitus, and the use of standardized protocols to measure risk factors and nephropathy, it also has some limitations. First, the small number of non-white participants may limit the ability to generalize our findings to other ethnic groups. Secondly, it is possible that selective survival (persons with higher levels of inflammatory and endothelial dysfunction who developed kidney disease were less likely to be seen in follow-up than persons who did not develop kidney disease due to higher mortality) resulted in underestimation of the relationship between these markers and the incidence of GP. Thirdly, we did not measure the total amount of protein in a 24-h urine collection test or measure the protein/creatinine ratio. Our measurement of proteinuria in a random sample using a reagent strip may have resulted in misclassification reducing our ability to detect associations of markers with proteinuria. However, a positive dipstick has been reported to have a sensitivity of $100 \%$ and a specificity of $82 \%$ (21), suggesting the bias to be small.

In summary, independent of blood pressure, glycemia, and other confounders, we found strong associations of some inflammatory and endothelial dysfunction markers with prevalent and incident GP in persons with type 1 diabetes mellitus. It remains to be seen if reduction in inflammation would have a beneficial effect on the incidence of nephropathy in persons with type 1 diabetes mellitus.

\section{Declaration of interest}

The authors declare that there is no conflict of interest that could be perceived as prejudicing the impartiality of the research reported.

\section{Funding}

This work was supported by the National Institutes of Health, Bethesda, Maryland, USA (number EY016379, to R Klein and B E K Klein; number DK073217, to R Klein); and by the American Diabetes Association, Alexandria, Virginia, USA (Mentor-Based Postdoctoral Fellowship Award, to R Klein). 


\section{References}

1 Parving HH, Hovind P, Rossing K \& Andersen S. Evolving strategies for renoprotection: diabetic nephropathy. Current Opinion in Nephrology and Hypertension 200110 515-522.

2 Krempf M. Specifics of diabetic nephropathy, viewpoint in diabetology: trends of diabetic nephropathy in diabetes. Néphrologie \& Thérapeutique 20062 S190-S192.

3 Stitt AW, Jenkins AJ \& Cooper ME. Advanced glycation end products and diabetic complications. Expert Opinion on Investigational Drugs 200211 1205-1223.

4 Rossing P. Promotion, prediction and prevention of progression of nephropathy in type 1 diabetes mellitus. Diabetic Medicine 199815 900-919.

5 Navarro JF, Mora C, Maca M \& Garca J. Inflammatory parameters are independently associated with urinary albumin in type 2 diabetes mellitus. American Journal of Kidney Diseases 200342 53-61.

6 Kang ES, Kim HJ, Ahn CW, Park CW, Cha BS, Lim SK, Kim KR \& Lee HC. Relationship of serum high sensitivity C-reactive protein to metabolic syndrome and microvascular complications in type 2 diabetes. Diabetes Research and Clinical Practice 2005 69 151-159.

7 Astrup AS, Tarnow L, Pietraszek L, Schalkwijk CG, Stehouwer CD, Parving HH \& Rossing P. Markers of endothelial dysfunction and inflammation in type 1 diabetic patients with or without diabetic nephropathy followed for 10 years: association with mortality and decline of glomerular filtration rate. Diabetes Care 200831 1170-1176.

8 Lin J, Glynn RJ, Rifai N, Manson JE, Ridker PM, Nathan DM \& Schaumberg DA. Inflammation and progressive nephropathy in type 1 diabetes in the diabetes control and complications trial. Diabetes Care 200831 2338-2343.

9 Klein R, Klein BE, Moss SE, DeMets DL, Kaufman I \& Voss PS. Prevalence of diabetes mellitus in Southern Wisconsin. American Journal of Epidemiology 1984119 54-61.

10 Klein R, Klein BE, Moss SE, Davis MD \& DeMets DL. The Wisconsin Epidemiologic Study of Diabetic Retinopathy. II. Prevalence and risk of diabetic retinopathy when age at diagnosis is less than 30 years. Archives of Ophthalmology 1984102 520-526.

11 Klein R, Klein BE, Moss SE \& Cruickshanks KJ. The Wisconsin Epidemiologic Study of Diabetic Retinopathy. XIV. Ten-year incidence and progression of diabetic retinopathy. Archives of Ophthalmology 1994112 1217-1228.
12 Klein R, Knudtson MD, Lee KE, Gangnon R \& Klein BE. The Wisconsin Epidemiologic Study of Diabetic Retinopathy: XXII the twenty-five-year progression of retinopathy in persons with type 1 diabetes. Ophthalmology 2008115 1859-1868.

13 Klein R, Klein BE, Moss SE, Davis MD \& DeMets DL. The Wisconsin Epidemiologic Study of Diabetic Retinopathy. IX. Four-year incidence and progression of diabetic retinopathy when age at diagnosis is less than 30 years. Archives of Ophthalmology 1989 $107237-243$.

14 Mysliwiec M, Balcerska A, Zorena K, Mysliwska J, Nowacka M, Lipowski P \& Raczynska K. Selected immunologic and biochemical risk factors of the retinopathy and nephropathy development in children with diabetes mellitus type 1. Endokrynologia, Diabetologia i Choroby Przemiany Materii Wieku Rozwojowego 200612 269-273.

15 Stehouwer CD, Gall MA, Twisk JW, Knudsen E, Emeis JJ \& Parving HH. Increased urinary albumin excretion, endothelial dysfunction, and chronic low-grade inflammation in type 2 diabetes: progressive, interrelated, and independently associated with risk of death. Diabetes 200251 1157-1165.

16 Nakahara C, Kanemoto K, Saito N, Oyake Y, Kamoda T, Nagata M \& Matsui A. C-reactive protein frequently localizes in the kidney in glomerular diseases. Clinical Nephrology 200155 365-370.

17 Schwedler SB, Guderian F, Dammrich J, Potempa LA \& Wanner C. Tubular staining of modified C-reactive protein in diabetic chronic kidney disease. Nephrology, Dialysis, Transplantation 200318 2300-2307.

18 Wotherspoon F, Laight DW, Browne DL, Turner C, Meeking DR, Allard SE, Munday LJ, Shaw KM \& Cummings MH. Plasma homocysteine, oxidative stress and endothelial function in patients with type 1 diabetes mellitus and microalbuminuria. Diabetic Medicine 200623 1350-1356.

19 Hofmann MA, Kohl B, Zumbach MS, Borcea V, Bierhaus A, Henkels M, Amiral J, Schmidt AM, Fiehn W, Ziegler R, Wahl P \& Nawroth PP. Hyperhomocyst(e)inemia and endothelial dysfunction in IDDM. Diabetes Care 199821 841-848.

20 Unlucerci Y, Bekpinar S, Gurdol F \& Seferoglu G. A study on the relationship between homocysteine and diabetic nephropathy in rats. Pharmacological Research 200245 249-252.

21 Zelmanovitz T, Gross JL, Oliveira J \& de Azevedo MJ. Proteinuria is still useful for the screening and diagnosis of overt diabetic nephropathy. Diabetes Care 199821 1076-1079.

Received 19 February 2010

Accepted 22 March 2010 